Methods We searched the electronic databases of Medline, Embase and PsycINFO for articles published between 2008 and 12 August 2019 and included the references of a systematic review performed for the period before 2008. Primary longitudinal studies were included when outcome data were described in terms of SRD assessment or a dichotomous outcome, based on a validated questionnaire, and at least two levels of work-related exposure were reported. The selection of the work-related risk factors in the meta-analyses was based on: (1) sufficient contrast between reported exposure categories; (2) effect estimates controlled for other non-work-related factors; 3) homogeneity in definition or measurement of risk factors. The quality of evidence was assessed using the Grades of Recommendations, Assessment, Development and Evaluation framework.

Result Seventeen studies met the inclusion criteria. The metaanalysis revealed moderate evidence for associations between SRDs and effort reward imbalance $(\mathrm{OR}=1.9$, 95\% CI 1.70 2.15), high job demands $(\mathrm{OR}=1.6,95 \%$ CI 1.41- 1.72), organisational justice (OR's 1.6 to 1.7 , CI's 1.44-1.86), social support (OR's 1.3 to 1.4, CI's 1.16-1.69), high emotional demands $(\mathrm{OR}=1.6,95 \% \mathrm{CI} 1.35-1.84)$, and decision authority $(\mathrm{OR}=1.3$, CI 1.20-1.49). No significant or inconsistent associations were found for job insecurity, decision latitude, skill discretion and bullying.

Conclusion Moderate evidence was found that work-related psychosocial risk factors are associated with a higher risk of SRDs. Effort-reward imbalance, low organisational justice and high job demands exhibited the largest increased risk of SRDs, varying from 60 to $90 \%$.

\section{0-218 COGNITIVE IMPAIRMENT AND WORK STATUS AMONG PATIENTS WITH WORK-RELATED STRESS: VALIDATION OF AN OBJECTIVE COGNITIVE SCREENER}

'Johan Høy Jensen, Kamilla W Miskowiak, Scot E Purdon, Esben Meulengracht Flachs, Maja S Worm, Jane Froelund Thomsen, Nanna H Eller. 'Bispebjerg and Frederiksberg Hospital, Denmark

\subsection{6/OEM-2021-EPI.157}

Introduction Many individuals with long-term work-related stress complain that cognitive limitations impede their vocational functioning. Subjective cognitive complaints, however, may not accurately reflect objective cognitive impairment.

Objectives We aimed to evaluate objective and subjective tools for screening of neurocognitive impairment and assess cognitive functioning as a determinant for subsequent work status among patients with work-related stress.

Methods 82 patients with work-related stress participated in a $1 \frac{1}{2}$-hour assessment of demographics, objective and subjective cognitive status, and psychosocial functioning. Objective measures included the Screen for Cognitive impairment in Psychiatry, Danish version, (SCIP-D) comprising five cognitive subtests (administration $<20$ min.) and standardized neuropsychological tests that tapped into the same cognitive domains. Subjective cognitive impairment was assessed with the Cognitive Failure Questionnaire. Work status was evaluated by telephone 6 months later. A total of 78 patients were matched to preexisting norm data from 79 healthy controls using bootstrapping with 1000 resamples according to age, sex, and estimated premorbid intelligence $(\mathrm{N}=78000)$.
Results Strong correlations with all standardized neuropsychological tests indicated the concurrent validity of the SCIP-D (Total Score: $r=0.76, p$ ). There was no correlation between objective and subjective measures of cognitive status (Pearson's r0.30). Based on logistic Receiver-Operating-Characteristic analysis, the optimal SCIP-D Total-score cut-off was $\leq 72$, which identified $43.2 \%$ of the patients with global objective cognitive impairment (AUC $=0.84,95 \% \mathrm{CI}=0.76-0.93$, Sensitivity $=0.77$, Specificity $=0.73)$. Each one-unit increase in the objective SCIP-D Total score (mean $=74.2$, SD $=9.7$ ) was associated with a higher odds ratio of 1.12 (adjusted 95\% $\mathrm{CI}=1.004-1.253$ ) for employment at 6-month follow-up relative to full time sick-leave. Subjective cognitive impairment was not associated with subsequent work status.

Conclusion The SCIP-D was a valid objective cognitive screener predicting employment 6 months later among patients with work-related stress. The SCIP-D offers brief assessment of performance-based cognitive skills for use in occupational clinics and epidemiological research.

\section{0-226 CONTRIBUTORS TO STRESS AND BURNOUT IN JUNIOR DOCTORS DURING THE COVID-19 PANDEMIC}

${ }^{1}$ Anli Yue Zhou, Maria Panagioti, Mark Hann, Peter Bower, Mumtaz Patel, Martie van Tongeren, Aneez Esmail, Raymond Agius. 'University of Manchester, United Kingdom

\subsection{6/OEM-2021-EPI.158}

Introduction Junior doctors have previously reported high levels of burnout; and additional stressors have likely emerged from the COVID-19 pandemic may further accelerate burnout. There is a need to identify which particular stressors are most likely to lead to burnout in junior doctors in order to develop appropriate interventions.

Objectives 1) To develop a comprehensive list of stressors that are relevant to junior doctors, which includes general work and non-work related stressors as well as stressors that have specifically emerged with the pandemic. 2) To assess which of these stressors are most strongly associated with burnout.

Methods An anonymous online questionnaire was sent to 1000 randomly selected junior doctors in the North West of England between $10 / 07 / 20$ to $04 / 08 / 20$. It included 37 questions on general and pandemic-specific stressors, and the Maslach Burnout Inventory (MBI) Health Services Survey. Stepwise regression analyses were undertaken to assess associations between stressors and burnout.

Results In total, 326 responses were collected (response rate $=33 \%$ ). Of the top 10 stressors rated by junior doctors, $60 \%$ were related to the pandemic. Multiple stressors were found to be associated with the burnout dimensions. Fatigue $(\beta=0.43)$, pandemic-related workload increase $(\beta=0.33)$ and feeling isolated $(\beta=0.24)$ had the strongest associations with Emotional Exhaustion, whereas fatigue $(\beta=0.24)$, uncertainty around COVID-19 information $(\beta=0.22)$ and doing unproductive tasks $(\beta=0.22)$ had the strongest associations with Depersonalisation.

Conclusion Junior doctors experience a combination of general stressors and additional stressors emerging from the pandemic which significantly to impact on burnout. Monitoring these stressors and targeting them as part of interventions could help mitigating burnout in junior doctors. 